\title{
The Role of Shell Model Nuclear Level Densities for Nuclear Astrophysics
}

\author{
Mihai Horoi* and Roman Senkov \\ Department of Physics, Central Michigan University, Mount Pleasant, Michigan 48859, USA \\ E-mail: horoi@phy.cmich.edu
}

\begin{abstract}
Precise estimation of the reaction rates necessary for an accurate prediction of stellar evolution heavily depends on the nuclear level density (NLD) model used in the calculation. In the last decade the most used reaction rates were those reported in Ref. [1], which are based on a refined version of the Fermi gas model to estimate the NLD. We developed new techniques based on nuclear statistical spectroscopy to calculate the spin and parity projected moments of the nuclear shell model Hamiltonian, which can be further used to obtain an accurate description of the nuclear level density up to about $12-15 \mathrm{MeV}$ excitation energy. In the last year we made some breakthroughs in our computational methodology, by using a proton-neutron formalism and by porting our codes to massively parallel computers, which allow us to increase the speed of our calculations by many orders of magnitude, offering the opportunity to calculate shell model NLDs for a much larger class of nuclei. The reaction rates for nuclei around the waiting point nucleus ${ }^{64} \mathrm{Ge}$ are calculated with our shell model NLDs and compared with other NLD models available in the reaction code talys [2].
\end{abstract}

11th Symposium on Nuclei in the Cosmos

19-23 July 2010

Heidelberg, Germany.

${ }^{*}$ Speaker. 
Precise estimation of the reaction rates necessary for an accurate prediction of stellar evolution heavily depends on the nuclear level density model (NLD) used in the calculation. In the last decade the most used reaction rates were those reported in Ref. [1], which are based on a refined version of the Fermi gas model to estimate the NLD. In recent years open codes, such as talys [2], became available, which can be used to compare the reaction rates calculated with different NLD models. The results provided by talys for the potentially rp-process waiting point nuclei ${ }^{64} \mathrm{Ge}$ and ${ }^{68} \mathrm{Se}$ show large variations $(>10)$ of their reaction rates when different NLD models are used at the temperatures relevant for the rp-process. In addition, these reaction rates are significantly different from those of Ref. [1] that are still mostly used in recent analyses [3]. Recently, we developed a methodology $[4,5,6,7,8,9,10]$ of calculating the spin and parity dependent shell model NLD, which is a very important ingredient in the Hauser-Feshbach theory for calculating reaction rates for nuclear astrophysics [1]. Our novel techniques based on nuclear statistical spectroscopy allow us to calculate the spin and parity projected moments of the nuclear shell model Hamiltonian that can be further used to obtain an accurate description of the nuclear level density up to about 12$15 \mathrm{MeV}$ excitation energy. In the last year we made some breakthroughs in our computational methodology, by implementing a proton-neutron formalism and by porting our codes to massively parallel computers [10], which allow us to increase the speed of our calculations by many orders of magnitude, thus offering the opportunity to calculate shell model NLDs for a much larger class of nuclei. With the new code one can obtain accurate spin- and parity-dependent NLDs in minutes for large valence spaces, making our method competitive with the alternative Monte Carlo based methods (see e.g. [11]).

The reaction rates for the waiting point nuclei ${ }^{64} \mathrm{Ge}$ and ${ }^{68} \mathrm{Se}$ are of large interest for a better understanding of the rp-process. The NLDs used in the Hauser-Feshbach codes that calculate crosssections and reaction rates are mostly based on the back shifted Fermi gas model (BSFG) [12] with some improvements that can approximate the spin and parity dependence [13]. This approach has the advantage of being amenable to fitting the existent experimental data, in particular the RIPL2 data base of neutron resonances [14]. Our algorithm for the spin- and parity-dependent first two moments of the shell model Hamiltonian is a major many-body microscopic approach that takes into account a reasonable mean-field and a realistic residual interaction among nucleons. Our recent analysis indicates that the shell model NLDs provide a better description of the data, especially for the low spins. Given the efficiency of the new algorithm that allows us to calculate very quickly the NLDs for a much larger class of nuclei, we could calculate some reaction rates for nuclei around ${ }^{64} \mathrm{Ge}$, which is suspected of being a waiting-point nucleus in the rp-process path. The valence space used to describe these nuclei consists of the $0 f_{5 / 2}, 1 p_{3 / 2}, 1 p_{1 / 2}$, and $0 g_{9 / 2}$ spherical orbits, permitting the description of NLDs of both parities. We used the JJ4PNA effective interaction, which was briefly described in Ref. [15]. For the reaction rates we used the nuclear reaction code talys [2].

The talys code has the advantage that its source is freely available and it can be checked by the community. It has implemented a large number of reaction models with their associated parameters that can be further tuned for a realistic comparison of different approaches. In particular, it has implemented five NLD models that depend on a number of standard parameters. The simplest model available, designated as M1 here, is based on constant temperature Fermi gas, and a backshifted Fermi gas model is also available. The most microscopic NLD models available are based 


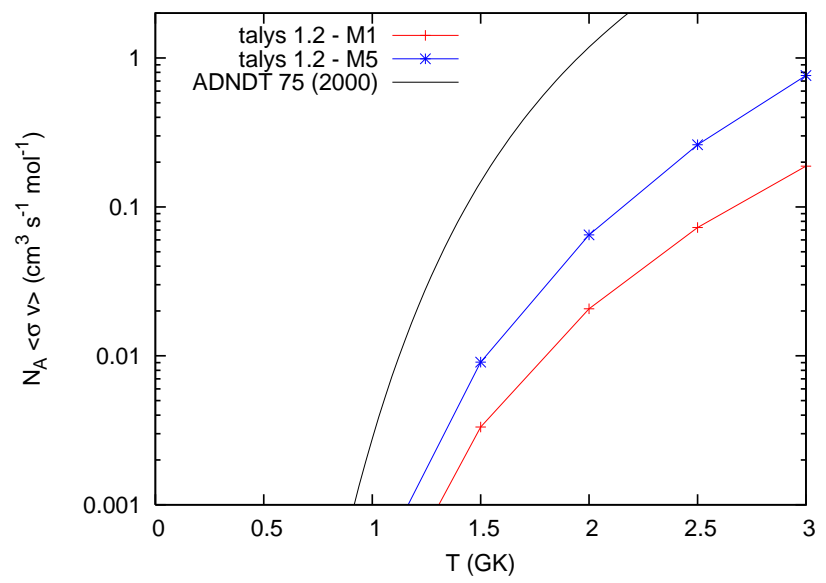

Figure 1: ${ }^{64} \mathrm{Ge}$ reaction rate calculated with two model for nuclear level density, M1 and M5 (see text for details), compared with that of Ref. [1] (ADNDT).

on the HF+BCS model (M4) of Ref. [16] and the HFB plus combinatorics model (M5) of Refs. $[17,18]$. The last model provides spin- and parity-dependent NLDs, which can also be found in tables at [20]. These are also the tables used by talys. This approach has the advantage that the tables can be modified to include any other tabulated spin- and parity-dependent NLDs. Our recent analysis [10] indicates that the M5 level densities significantly overshoot the shell model level densities, especially for the low spins of interest in low-energy reactions relevant for nuclear astrophysics. Therefore, we created an interface that replaces the low-energy part $(<12 \mathrm{MeV})$ of the NLDs in the M5 tables with our NLDs calculated with the moments method (Mom in Figs. 2,3) described above. The high energy part $(>12 \mathrm{MeV}$ ) was kept but rescaled to reflect the continuity of the NLD.

We first used the cross sections measured and analyzed in Ref. [19] as a test for the validity of the Hauser-Feshbach approach. Our talys calculations show generally reasonable good agreement with the data, in particular for the simple NLD model M1. The most microscopic NLD model M5 shows some discrepancies with the data, and the encouraging part is that when the M5 tables were modified with the shell model NLDs there was a clear improvement in the description of the data. The reaction rates at stellar temperatures depend significantly on the NLDs input to the reaction code. As an example, Fig. 1 shows a comparison of the (p,g) reaction rate for ${ }^{64} \mathrm{Ge}$ calculated with two NLD models, M1 and M5, and that of Ref. [1] (ADNDT 75 (2000)). One can see that there can be a factor of up to 50 between the results of different models. One should also mention that the $(\mathrm{p}, \mathrm{g})$ reaction rate is also sensitive to the model used for the gamma-ray strength function. Here we used the standard Brink-Axel Lorentzian [2]. All other parameters considered were the default talys choice. This large variations suggest that one should try to improve the consistency of the models used in the calculation of the reaction rates.

To further investigate the effects of NLDs on the reaction rates around the rp-process nucleus ${ }^{64} \mathrm{Ge}$, we show the (p,g) and (p,p) reaction rates for ${ }^{64} \mathrm{Ge}$ in Fig. 2, and those for ${ }^{65} \mathrm{As}$ in Fig. 3. The results for the microscopic model M5 and those for the NLDs extracted from the our moments method (Mom) are compared in these figures. One can see that while the reaction rates for the 


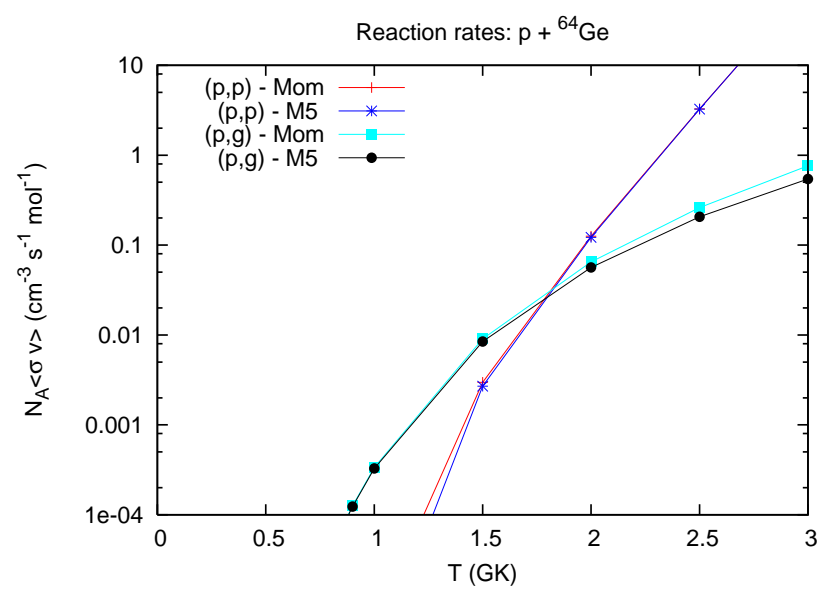

Figure 2: Comparison of reaction rates for ${ }^{64} \mathrm{Ge}$ calculated with NLDs given by the microscopic model of Hilaire (M5) and the moments method (Mom).

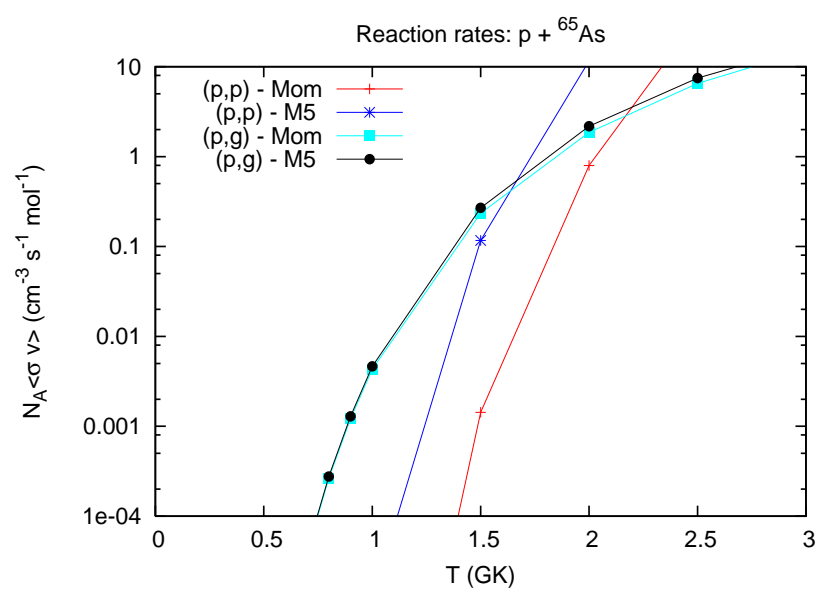

Figure 3: Comparison of reaction rates for ${ }^{65} \mathrm{As}$ calculated with NLDs given by the microscopic model of Hilaire (M5) and the moments method (Mom).

${ }^{64} \mathrm{Ge}$ are only mildly affected by the NLD model used, the (p,p) reaction rate for ${ }^{65}$ As changes by a factor of about 80 . While the direct relevance of these results has to be assessed in a network calculation, it is important to stress that two highly sophisticated microscopic NLD models could provide dramatically different conclusion about the reaction rates at relevant stellar temperature. A similar analysis for the reactions rates around the waiting-point nucleus ${ }^{68} \mathrm{Se}$ will be presented elsewhere.

In summary, we used our newly developed high-performance algorithm to calculate spin- and parity-dependent nuclear level densities for few nuclei around the rp-process nucleus ${ }^{64} \mathrm{Ge}$. We used these NLDs and the nuclear reaction code talys to calculate for the first time reaction rates relevant for the rp-procees around this nucleus. Our calculations show large variations in the results given by different NLDs while all other parameters are not changed. We conclude that more 
accurate NLDs are necessary, in addition to other important informations such as Q-values and life-times, in order to improve the quality of the predicted reaction rates at stellar temperatures. The implications of these results on network calculations remain to be assessed.

\section{Acknowledgments}

The authors would like to acknowledge the DOE UNEDF grant DE-FC02-09ER41584 for support. M.H. acknowledges support from the NSF grant PHY-0758099.

\section{References}

[1] Th. Rauscher, F.-K. Thielemann, At. Data. Nucl. Data. Tables 75, 1 (2000).

[2] talys 1.2 code, available at www.talys.eu.

[3] P. Schury et al., Phys. Rev. C 75, 055801 (2007).

[4] M. Horoi, J. Kaiser and V. Zelevinsky, Phys. Rev. C 67, 054309 (2003).

[5] M. Horoi, M. Ghita and V. Zelevinsky, Phys. Rev. C 69, 041307(R) (2004).

[6] M. Horoi, M. Ghita and V. Zelevinsky, Nucl. Phys. A 785C, 142 (2005).

[7] Mihai Horoi and Vladimir Zelevinsky, Phys. Rev. Lett. 98, 262503 (2007).

[8] M. Horoi, Proceedings of the International Symposium on Nuclear Astrophysics "Nuclei in the Cosmos - IX", CERN, Geneva, June 25-30, 2006. Proceedings of Science, PoS(NIC-IX)120, (2006), http://pos.sissa.it/.

[9] M. Scott and M. Horoi, Proceedings of the International Symposium on Nuclear Astrophysics "Nuclei in the Cosmos - X", Mackinac Island, July 25 - August 1, 2008, Proceedings of Science, PoS(NIC-X)132, (2008), http://pos.sissa.it/.

[10] R. Senkov and M. Horoi, Phys. Rev. C 82, 024304 (2010).

[11] Y. Alhassid, S. Liu, and H. Nakada, Phys. Rev. Lett. 99, 162504 (2007).

[12] T. Rauscher, F.-K. Thielemann, and K.-L. Kratz, Phys. Rev. C 56, 1613 (1997).

[13] D. Mocelj, T. Rauscher, G. MartŠnez-Pinedo, K. Langanke, L. Pacearescu, A. Faessler, F.-K. Thielemann, and Y. Alhassid, Phys. Rev. C 75, 045805 (2007).

[14] http://www-nds.iaea.org/ripl2/.

[15] J. Lee, M. B. Tsang, and W. G. Lynch, M. Horoi, and S.C. Su, Phys. Rev. C 79, 054611 (2009).

[16] P. Demetriou and S. Goriely, Nucl. Phys. A695, 95 (2001).

[17] S. Hilaire and S. Goriely, Nucl. Phys. A779, 63 (2006).

[18] S. Goriely, S. Hilaire, and A. J. Koning, Phys. Rev. C 78, 064307 (2008).

[19] A. V. Voinov, S. M. Grimes, C. R. Brune, M. J. Hornish, T. N. Massey, and A. Salas, Phys. Rev. C 76, 044602 (2007).

[20] http://www-astro.ulb.ac.be/Html/nld_comb.html . 